\title{
Occludin Regulates Actin Cytoskeleton in Endothelial Cells
}

\author{
Hiroki Kuwabara $^{1,2}$, Yasuo Kokai ${ }^{*}$, Takashi Kojima ${ }^{1}$, Reiko Takakuwa ${ }^{1}$, Michio Mori ${ }^{1}$, and Norimasa \\ Sawada ${ }^{1}$ \\ Department of ${ }^{1}$ Pathology and ${ }^{2}$ Orthopedic Surgery, Sapporo Medical University School of Medicine, S1W17, \\ Chuo-ku, Sapporo 060-8556, Japan
}

\begin{abstract}
Occludin is a major membrane component of tight junctions of endothelial cells, though the role of this molecule is not fully understood. RLE cells, derived from rat lung endothelial cells, express a negligible level of occludin with clear expression of E-cadherin and ZO-1 at cell junctions. Introduction of occludin by transfection induced clear junctional expression of occludin with few or no changes of expression of E-cadherin and ZO-1. The paracellular barrier function, as determined by transelectrical resistance and flux of non-ionic small molecules, was not detectably upregulated. When cells expressing occludin were cocultured with RLE cells null for occludin, clear junctional expression of occludin was observed irrespective of the expression of occludin on the apposing cells. Cortical actin was developed at the site of these occludin positive cell junctions. Treatment of cells with an actin depolymerizing agent, mycalolide B, abolished junctional expression of occludin together with E-cadherin and circumferential actin. ZO-1 showed relative resistance to this actin depolymerizing treatment and was maintained at the cell junctions, though fragmentation of immunoreactivity was detectable. Collectively, junctional expression of occludin was not associated with paracellular barrier function in this cell line. There was, however, a close correlation of occludin with the actin cytoskeleton, indicating a role of occludin as an important molecule in the regulation of the actin cytoskeleton in endothelial cells.
\end{abstract}

Key words: occludin/endothelial cell/junctional expression/cell-cell contact/tight junction

Occludin is a transmembrane molecule localized at tight junctions (Ando-Akatsuka et al., 1996; Fruse et al., 1992; Saitou et al., 1997). Initial studies of the epithelial system indicated that this molecule is important in the regulation of not only paracellular permeability but also the fence function of tight junctions (Balda et al., 1996; Chen et al., 1997; McCarthy et al., 1996). Occludin is abundant in endothelial cells, like those of brain capillaries, and is thought to be important for functions of the tight junction in these cells (Hirase et al., 1997). These model endothelial cells express a tight paracellular barrier upon addition of cAMP in combination with astrocyte conditioned medium (Rubin et al., 1991). Glial cell line-derived neurotrophic factor is suggested to be important for this activity in the astrocyte

\footnotetext{
* To whom correspondence should be addressed: Department of Pathology, Sapporo Medical University School of Medicine, S1W17, Chuo-ku, Sapporo 060-8556, Japan.

Tel: +81-11-611-2111 (ext. 2701), Fax: +81-613-5665

E-mail: yasokoki@samped.ac.jp

Abbreviations: FCS, fetal calf serum; CLSM, confocal laser scanning microscopy; PBS, phosphate-buffered saline; DMEM, Dulbecco's modified Eagle medium; MB, mycalolide B.
}

conditioned medium (Igarashi et al., 1999). It was, however, shown that lysophosphatidic acid reduces the paracellular barrier function, but the expression of occludin is still observed in a manner comparable to that in endothelial cells with a tight paracellular barrier (Schulze et al., 1997).

Tight junctions localize at the apicalmost surface of apposing cells and serve to regulate the passage of water, ions and small molecules through the paracellular space. It has also been suggested that the tight junction is closely associated with the actin cytoskeleton in terms of both structure and function (Balda and Matter, 1998; Goodenough, 1999). Recent molecular studies reported that the $\mathrm{COOH}$-terminal domain plays a pivotal role for binding to $\mathrm{ZO}-1$ and, in turn, ZO-1 binds directly to actin (Fanning et al., 1998; Furuse et al., 1994; Mitic et al., 1999). We previously reported that expression of occludin in the rodent endothelial cell line, designated RLE, modulates the actin cytoskeleton and caused the development of fine cortical actin bundles at the cell border (Atsumi et al., 1999).

In the present report we studied cellular factors that 
require junctional expression of occludin in this cell line in relation to paracellular barrier function. The study revealed that junctional expression of occludin is dependent on cell-cell interaction and is closely associated with the actin cytoskeleton. Furthermore, junctional expression of occludin was clearly observed at the junctions between cells expressing occludin and cells lacking occludin. This observation indicates that localization of occludin is strictly regulated by the cytoplasmic components. Taken together, the present observations suggest that occludin plays an important role in tight junctions in close association with the actin cytoskeleton in endothelial cells.

\section{Materials and Methods}

\section{Reagents}

All reagents were purchased from Sigma (St. Louis, MO) unless otherwise stated.

\section{Cell culture and tests for paracellular barrier functions}

Cells were routinely maintained in DMEM containing with $10 \%$ FCS. To study paracellular barrier functions, $2 \times 10^{5}$ cells were cultured in 12-well Transwell culture filter (Coster, Cambridge, UK) (wells $1 \mathrm{~cm}$ in diameter). These cells were cultured for 3 to 4 days to reach confluent condition. Under the confluence, paracellular fluxes of inulin, mannitol and dextran was measured as described (Satoh et al., 1996). Transelectrical resistance was measured every two days after inoculation till the 10th culture day. The result was expressed as a mean of six sets of cultures from three independent experiments. The SD in all these experiments was less than $7 \%$, and thus is deleted from Figure 2.

\section{Plasmids for cell transfection}

pEF-BOS (a generous gift from Dr. S. Nagata of Osaka University) (Mizushima and Nagata, 1990) was inserted at the XbaI site with the full coding sequence of rat occludin cDNA tagged with the FLAG epitope by PCR at the $\mathrm{C}$ terminus. Primers used were: S128: 5'GCTCTAGAGCCATGTCTGTGAGGCCTTTTGAG3' S243: 5'TCTAGACTACTTGTCATCGTCGTCCTTGTAGTCGGTTTTCCGT CTGTCATAGTC3' for the pBOS/oc.FLAG. All PCR products and subcloning junctions were verified by sequencing. These plasmids were transfected as described (Atsumi et al., 1999). Several independent clones were selected by occludin expression was determined by immunocytochemistry. Since all the clones selected gave virtually identical results, we present data from one clone, designated RLE/oc.flag, as a representative. pEGFP plasmid (Ivitrogen, Netherlands) was transfected to cells to express green fluorescent protein transiently by Lipofectamin 2000 (Gibco/BRL, Tokyo, Japan) per manufacture's instruction.

\section{Mycalolide B treatment}

Mycalolide B (Saito et al., 1994) was purchased Wako Chemicals
(Osaka, Japan) and dissolved at $1 \mathrm{mM}$ in dimethyl sulfoamide and then diluted to $2 \mu \mathrm{M}$ with DMEM containing 10\% FCS (Morita et al., 1999).

\section{Immunocytochemistry and immunoblotting}

These were performed as described (Atsumi et al., 1999). Antibodies used were rabbit anti-occludin (developed against the C-terminus of rat occludin in our laboratory)(Atsumi et al., 1999), rabbit anti-ZO-1 (Zymed, San Francisco, CA), mouse anti-E cadherin (Transduction Lab., Lexington, KY) and anti-FLAG M2 monoclonal antibody at the dilutions recommended by the manufacturers. Confocal images were obtained with a MRC-1024 CLSM (Biorad, Tokyo, Japan).

\section{Results}

\section{Occludin localized at cell junctions when expressed by} transfection in RLE cells

The endothelial cell line RLE expressed barely detectable levels of occludin as studied by immunoblotting and immunocytochemistry (Fig. 1A) with clear expression of both Ecadherin and ZO-1 at cell borders. When RLE cells were transfected with pBOS/oc.FLAG, clear expression of occludin was observed at cell junctions of these cells (Fig. 1B). Immunoblotting also showed a single molecular mass with the expected size (see inset in Fig. 1A). The expression of E-cadherin and ZO-1 was comparable in wild-type RLE cells and occludin-expressing cells (Fig. 1C to F).

\section{Paracellular barrier functions were not altered by expression of occludin}

The RLE/oc.flag cells were cultured in Transwell culture filter and various parameters for paracellular barrier functions were compared to those of the wild-type RLE cells (Fig. 2) No significant differences were observed in any of the parameters studied, including TER (A) and paracellular fluxes of inulin (B), mannitol (C) and dextran (D).

\section{Occludin localized at cell-junctions of two apposing endothelial cells when one was null for occludin}

Wild-type RLE cells labeled by pEGFP and RLE cells expressing occludin (RLE/oc.flag) were mixed at a ratio of 2 to 1 and cultured for two days. To detect wild type RLE cells expressing no detectable occludin, they were labeled by green fluorescent proteins by transfection.

In the cell junctions of two apposing cells where one was null for occludin (see arrowheads in Fig. 3), occludin was found to localize at these cell junctions. The cell-junctions between occludin-positive cells were also positive for occludin (asterisks in Fig. 3), though cell borders lacking apposing cells showed no occludin expression (arrow in Fig. 3). 

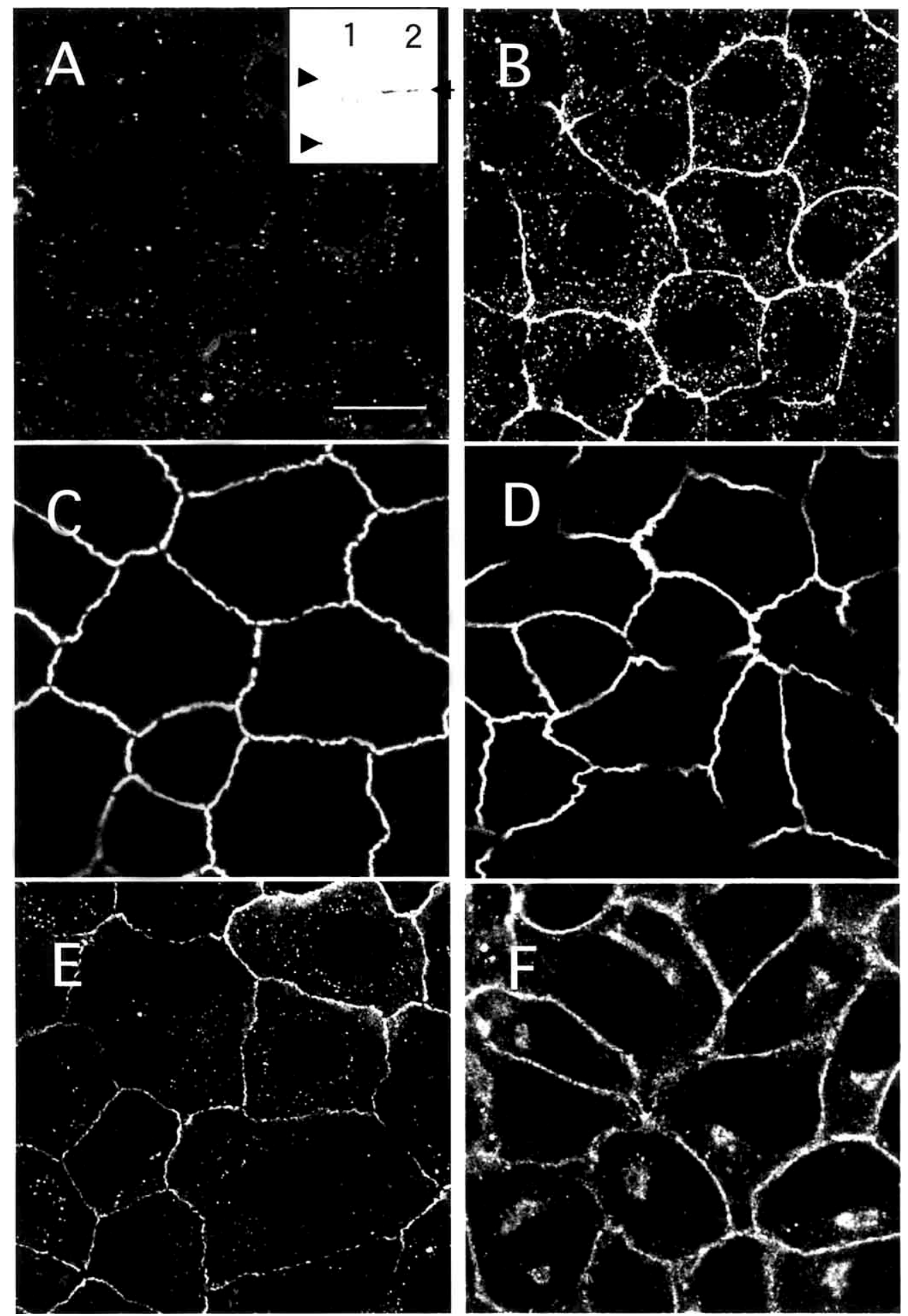

Fig. 1. Cell border expression of occludin in RLE transfectants. Wild-type RLE cells (A, C, E) and RLE cells expressing occludin (RLE/oc.flag) (B, D, F) were stained for occludin (A, B), ZO-1 (C, D) and E-cadherin (E, F). Clear junctional localization of occludin is observed in RLE/oc.flag but not in the wild-type RLE cells. Immunoblotting shows the expected molecular mass of occludin in RLE/oc.flag, though almost no expression was detected in the wild-type RLE cells (inset in A) (arrowhead indicates the molecular standard, upper; $76 \mathrm{Kd}$, Lower; $32 \mathrm{Kd}$ ). Occludin expression did not obviously change

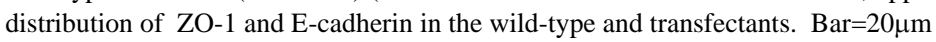




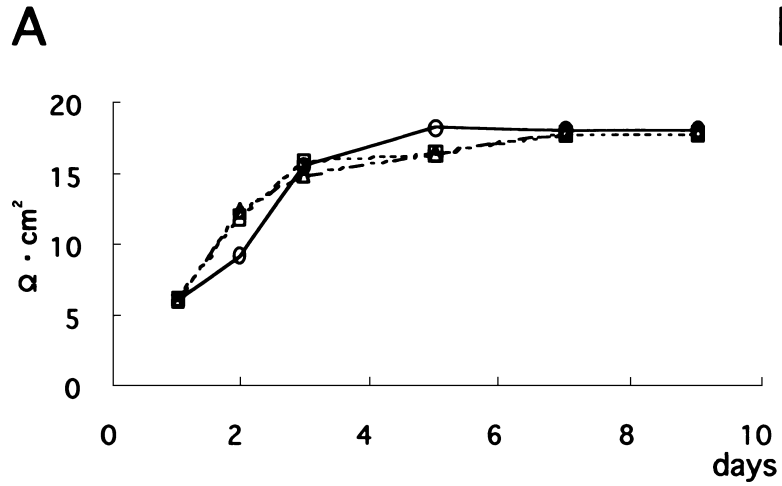

B
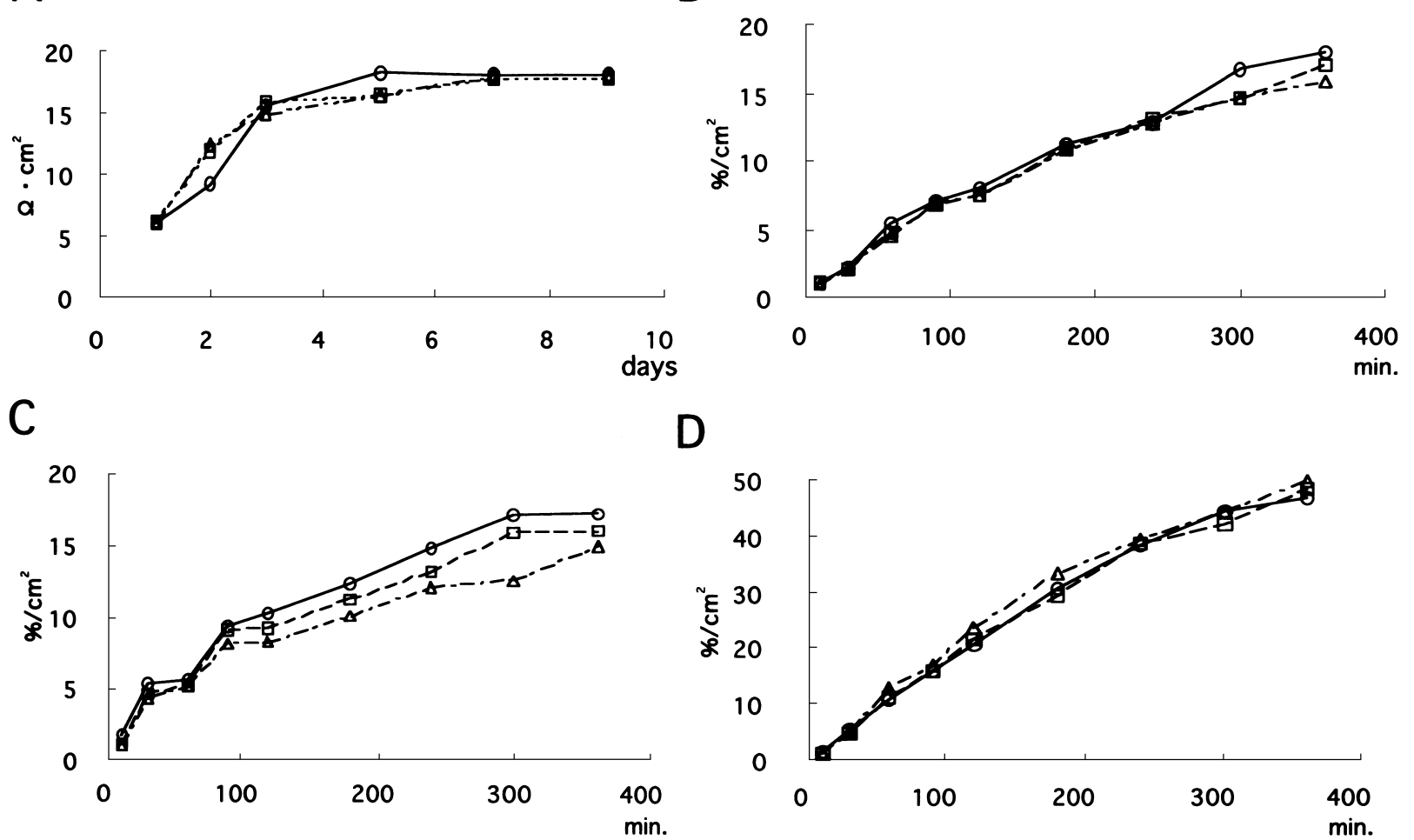

Fig. 2. Paracellular barrier functions are not altered by expression of occludin. RLE/oc.flag cells were cultured in Transwell culture filter and various parameters for paracellular barrier functions were compared to those of the wild-type RLE cells. For all the parameters studied, no significant changes were observed, including TER (A) and paracellular flux of inulin (B), mannitol (C) and dextran (D). Circles; wild-type RLE, Triangles; RLE cells expressing the neo gene only, Rectangles; RLE/oc.flag cells.

\section{Cell border expression of occludin closely correlated with fine circumferential actin bundles}

Wild-type RLE cells and RLE/oc. flag cells were mixed at a ratio of $5: 1$ and cultured for two days. These cells were stained for both FLAG and actin. As shown in Figure 4, occludin was found at cell junctions between two apposing cells where one was null for occludin, as well as at junctions between two occludin-positive cells (Fig. 4A). Actin staining by Texas red-phalloidin was found at the cell junctions and was quite similar to the staining pattern of occludin (compare Fig. 4 A and B). This is consistent with our previous study in which expression of occludin in RLE cells correlated with circumferential actin development (Lapierre et al., 1999).

\section{Disruption of actin cytoskeleton abolished cell-border expression of occludin, E-cadherin and cortical actin bundles}

To study the effect of actin depolymerization on the localization of occludin, RLE/oc.flag cells were cultured to confluence. Mycalolide B, an actin depolymerizing agent, was added to $2 \mu \mathrm{M}$ for $2 \mathrm{hr}$. Junctional localization of both occludin and E-cadherin was abolished with this treatment, together with the disappearance of the cortical actin (Fig. 5). Although the staining pattern of $\mathrm{ZO}-1$ became less sharp, ZO-1 was relatively resistant to this treatment. All of these changes were reversible within $24 \mathrm{hr}$ when cells were returned to the normal medium (ref. 26 and data not shown).

\section{Discussion}

We studied the role of occludin in endothelial cells using a cultured rat endothelial cell line designated RLE. This cell line lacked a detectable level of occludin, but had clear expression of E-cadherin and ZO-1 at its cell border. A freeze fracture study and RT-PCR of claudin 5 (Motita et al., 1999) revealed that RLE cells also lack both claudin 5 expression and typical tight junctional strands (data not shown). The lack of occludin expression, apparent lack of tight junctions, and the functional cadherin system enable us to study how occludin acts at cell junctions by using a transfection technique.

When occludin was expressed by transfection, it localized at cell junctions in a cell-cell contact-dependent manner. These occludin- expressing cells showed no changes of paracellular barrier functions as determined by TER and the flux of small molecules. By coculturing occludin-negative 

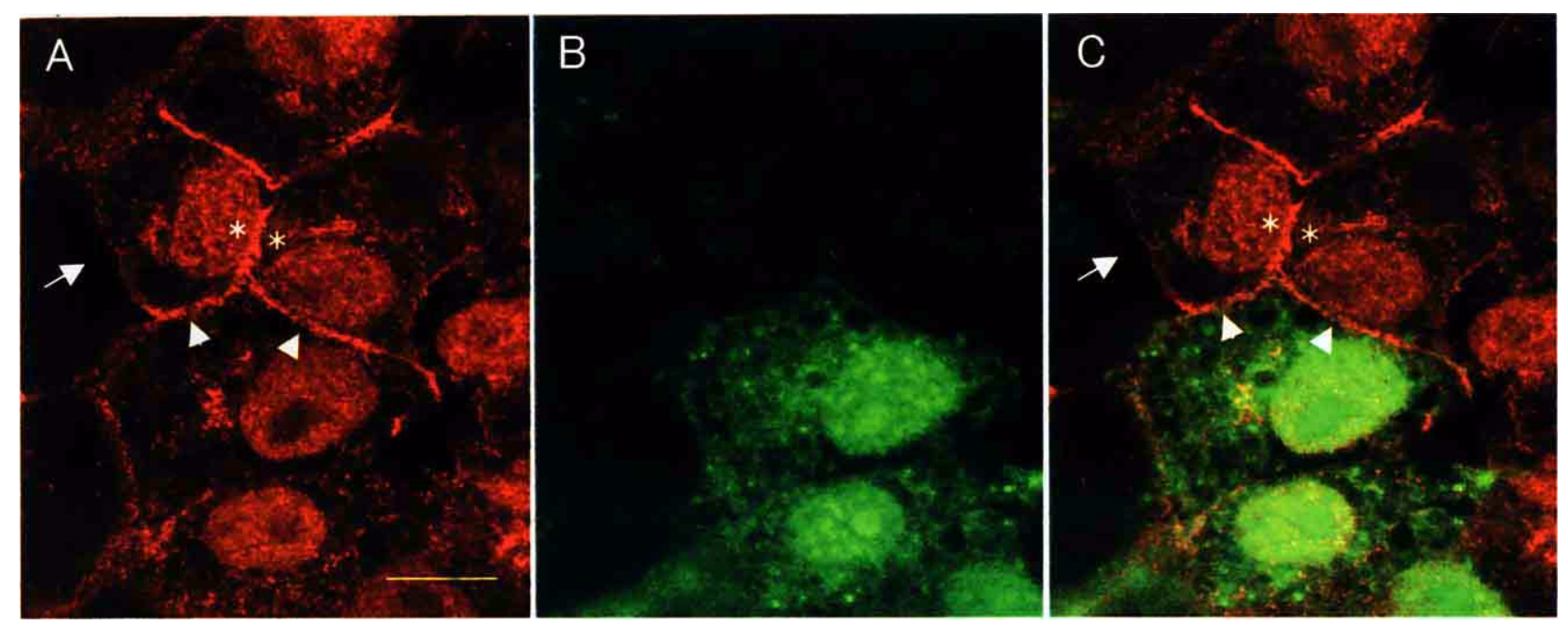

Fig. 3. Occludin localized at cell-junctions of two apposing cells where one is null for occludin. Wild-type RLE labeled by EGFP (B, C) and RLE/oc.flag cells were mixed at a ratio of $2: 1$ and cultured for two days. Cells were then stained by anti-FLAG antibody. Junctional expression of occludin is visible at the junction of two apposing cells where one is null for occludin (A,C, arrowhead). Junctions between two occludin expressing cells are also positive (A, C, asterisks), though junctions with no apposing cells are negative for fluorescent signals (A, C, arrows).
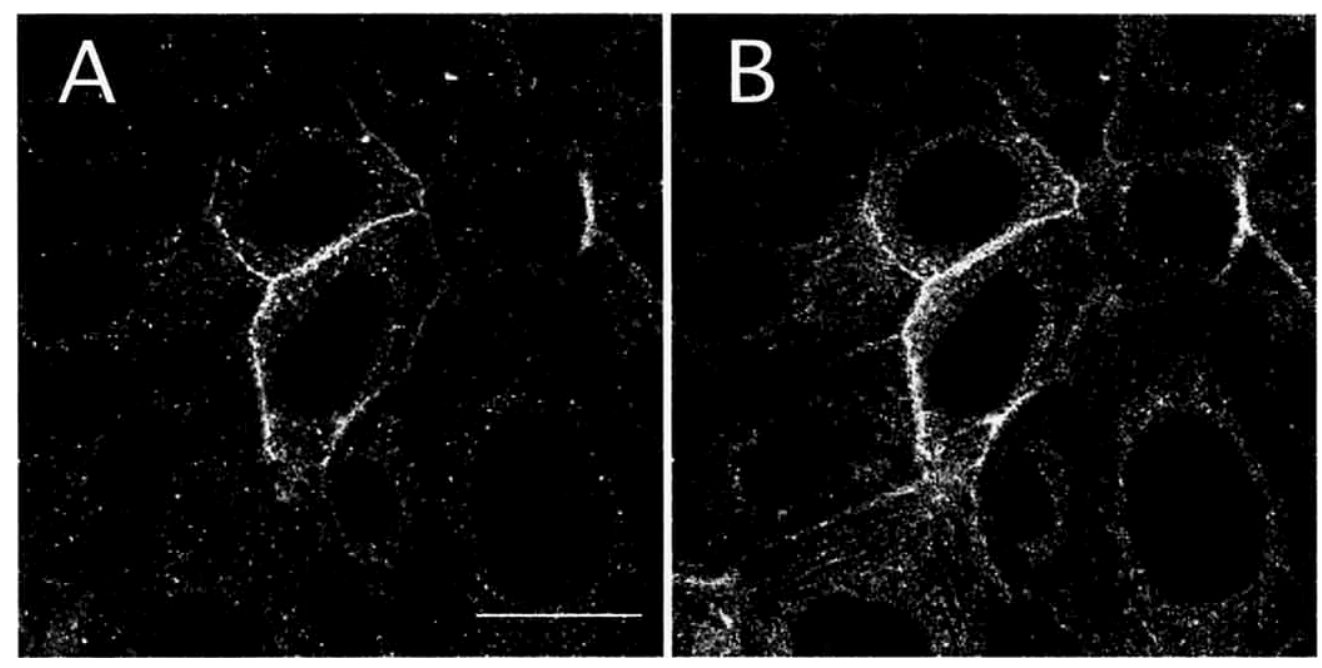

Fig. 4. Cell border expression of occludin closely correlates with fine circumferential actin bundles. Wild type RLE cells and RLE/oc.flag cells were cultured to confluence as in Figure 2 and stained for FLAG epitope (A) and actin (B). Occludin localizes again at cell junctions between two apposing cells where one is null for occludin. Fine cortical actin bundles are observed at the cell border, which is indistinguishable for the distribution of occludin. $\operatorname{Bar}=20 \mu \mathrm{m}$

wild RLE cells and occludin-expressing cells, expression of occludin in wild-type and occludin-positive cells was observed. There was also colocalization of occludin and fine cortical actin bundles at cell borders. Treatment of occludinpositive cells with the actin-depolymerization reagent, mycalolide B, abolished junctional localization of occludin and E-cadherin, but not that of ZO-1. It seems conceivable that junctional expression of occludin is closely associated with the actin cytoskeleton to anchor tight junctions rather than directly controlling the barrier function of the junction.
Using chimeric molecules of connexin and occludin, Mitic et al. revealed the molecular basis of occludin localization at tight junctions (Mitic et al., 1999). They showed that a connexin chimera containing the $\mathrm{COOH}$ terminal domain but not the proximal domain of occludin localized at tight junctions of MDCK cells and at cell junctions of NRK fibroblasts. Given that the importance of the $\mathrm{COOH}$ terminal of occludin is strongly linked to the binding ability to ZO-1, occludin seems to play a role in stabilizing these molecules at tight junctions to actin cytoskeleton. The binding 
H. Kuwabara et al.

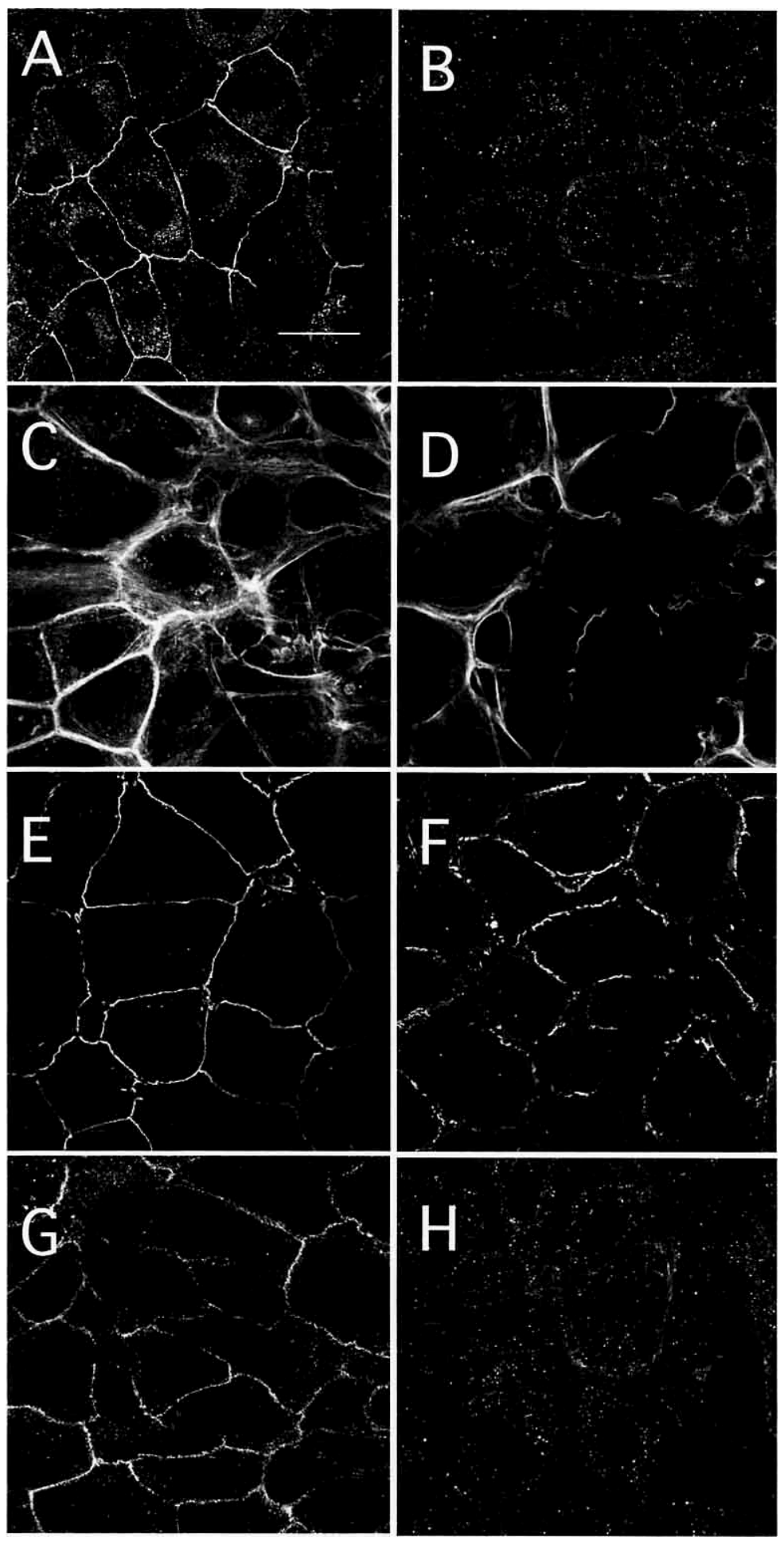

Fig. 5. Disruption of actin cytoskeleton abolishes cell-border expression of occludin, E-cadherin and cortical actin bundles. RLE/oc.flag cells were cultured to confluence and treated with medium alone (A, C, E, G) or medium containing $2 \mu \mathrm{M}$ mycalolide B (MB) (B, D, F, H) for 2 hr. Cells were stained for FLAG epitope (A, B), actin (C, D), ZO-1 (E, F) and E-cadherin (G, H). MB treatment abolished junctional expression of both occludin and E-cadherin, whereas ZO-1 stayed at cell junctions with fragmentation. Actin cytoskelton is disrupted in RLE/oc.flag cells treated with MB under these conditions (compare $\mathrm{D}$ to $\mathrm{C})$. Bar $=20 \mu \mathrm{m}$ 
capability of the extracellular domain of occludin was shown to be much lower than that of claudin (Kubota et al., 1999). The present study revealed that occludin could localize at cell contacts between two apposing cells when one was null for occludin. The C-terminal cytoplasmic domain of occludin is much larger than those of claudins, and is reported to have several domains that interact with other molecules (Fanning et al., 1998; Furuse et al., 1994; Haskins et al., 1998; Itoh et al., 1999; Lapierre et al., 1999). Thus, occludin might play a part in tight junction regulation by interactions with intracellular scaffold molecules rather than by interaction with occludin of apposing cells.

There is a growing body of evidence that suggests an important role of the actomyosin system in the regulation of tight junctions (Morita et al., 1999). It is, however, not fully understood how the actin cytoskeleton is involved in regulation of the structure or function of the tight junction and which molecule plays a central role for this particular linkage. Since occludin is a universal component of tight junctions and specific for this particular junction, the main role of occludin at tight junctions could be as a co-modulator of tight junction functions. We recently reported that fence and gate functions of MDCK cells could be differentially regulated by using optimum treatment with mycalolide B. During this uncoupling, occludin localized at sites compatible for the tight junction. Again, loss of barrier function did not correlate with the localization of occludin. Furthermore, treatment of rat hepatocytes in primary culture with mycalolide B abolished junctional expression of occludin (Kojima et al., 1999). Taken together, these results suggested that actin plays a pivotal role for regulating the fullblown tight junctions. In the present report, we dissected a part of this molecular complex and showed that occludin may be an important parameter linked closely to the structural maintenance of the actin cytoskeleton.

Acknowledgments. This work was supported in part by Grants-in-Aid from the Ministry of Education, Science, Culture, and Sports, and Health and Welfare of Japan and the Hokkaido Geriatrics Research Institute.

\section{References}

Ando-Akatsuka, Y., Saitou, M., Hirase, T., Kishi, M., Sakakibara, A., Itoh, M., Yonemura, S., Furuse, M., and Tsukita, S. 1996. Interspecies diversity of the occludin sequence: cDNA cloning of human, mouse, dog, and rat-kangaroo homologues. J. Cell Biol., 133: 43-47.

Atsumi, S-I., Kokai, Y., Tobioka, H., Kuwahara, K., Kuwabara, H., Takakuwa, T., Sasaki, K., Sawada, N., Mitaka, T., Mochizuki, Y., Imai, K., and Mori, M. 1999. Occludin modulates organization of perijunctional circumferential actin in rat endothelial cells. Med. Electron. Microsc., 32: 11-19.

Balda, M.S., Whitney, J.A., Flores, C., Gonzalez, S., Cereijido, M., and Matter, K. 1996. Functional dissociation of paracellular permeability and transepithelial electrical resistance and disruption of the apical-basolateral intramembrane diffusion barrier by expression of a mutant tight junction membrane protein. J. Cell Biol., 134: 1031-1049.

Balda, M.S. and Matter, K. 1998. Tight junctions. J. Cell Sci., 111: 541-
547.

Chen, Y-H., Merzdorf, C., Paul, D.L., and Goodenough, D.A. 1997. $\mathrm{COOH}$ terminus occludin is required for tight junction barrier function in early Xenopus embryos. J. Cell Biol., 138: 891-899.

Fanning, A.S., Jameson, B.J., Jesaitis, L.A., and Anderson, J.M. 1998. The tight junction protein ZO-1 establishes a link between the transmembrane protein occludin and the actin cytoskeleton. J. Biol. Chem., 273: 29745-29753.

Furuse, M., Hirase, T., Itoh, M., Nagafuchi, A., Yonemura, S., Tsukita, S., and Tsukita, S. 1992. Occludin: A novel integral membrane protein localizing at tight junctions. J. Cell Biol., 123: 1777-1788.

Furuse, M., Itoh, M., Hirase, T., Nagafuchi, A., Yonemura, S., Tsukita, S., and Tsukita, S. 1994. Direct association of occludin with ZO-1 and its possible involvement in the localization of occludin at tight junctions. J. Cell Biol., 127: 1617-1626.

Goodenough, D.A. 1999. Plugging the leaks. Proc. Natl. Acad. Sci. USA, 96: 319-321.

Haskins, J., Gu, J., Hibbard, J., and Stevenson, B.R. 1998. ZO-3, a novel member of the MAGUK protein family found at the tight junction, interacts with ZO-1 and occludin. J. Cell Biol., 141: 199-208.

Hirase, T., Staddon, J.M., Saitou, M., Ando-Akatsuka, Y., Itoh, M., Furuse, M., Fujimoto, K., Tsukita, S., and Rubin, L.L. 1997. Occludin as a possible determinant of tight junction permeability in endothelial cells. $J$. Cell Sci., 110: 1603-1613.

Igarashi, Y., Utsumi, H., Chiba, H., Yamada-Sasamori, Y., Tobioka, H., Kamimura, Y., Furuuchi, K., Kokai, Y., Nakagawa, T., Mori, M., and Sawada, N. 1999. Glial cell line-derived neurotrophic factor induces barrier function of endothelial cells forming the blood-brain barrier. Biochem. Biophysics. Res. Com., 261: 108-112.

Itoh, M., Morita, K., Tsukita, S. 1999. Characterization of ZO-2 as a MAGUK family member associated with tight as well as adherens junctions with a binding affinity to occludin and a-catenin. J. Biol. Chem., 274: 5981-5986.

Kojima, T., Sawada, N., Yamamoto, M., Kokai, Y., Mori, M., and Mochizuki, Y. 1999. Disruption of circumferential actin filament causes disappearance of occludin from the cell borders of rat hepatocytes in primary culture without distinct changes of tight junction strands. Cell Struct. Funct., 24: 11-17.

Kubota, K., Furuse, M., Sasaki, H., Sonoda, N., Fujita, K., Nagafuchi, A., and Tsukita, S. 1999. $\mathrm{Ca}\left({ }^{2+}\right)$-independent cell-adhesion activity of claudins, a family of integral membrane proteins localized at tight junctions. Curr. Biol., 23: 1035-1038.

Lapierre, L.A., Tuma, P.L., Navarre, J., Goldenring, J.R., and Anderson, J.M. 1999. VAP-33 localizes to both an intracellular vesicle population and with occludin at the tight junction. J. Cell Sci., 112: 3723-3732.

McCarthy, K.M., Skare, I.B., Stankewich, M.C., Furuse, M., Tsukita, S., Rogers, R.A., Lynch, R.D., and Schneeberger, E.E. 1996. Occludin is a functional component of the tight junction. J. Cell Sci., 109: 2287-2298.

Mitic, L.L., Schneeberger, E.E., Fanning, A.S., and Anderson, J.M. 1999. Connexin-occludin chimeras containing the ZO-binding domain of occludin localize at MDCK tight junctions and NRK cell contacts. $J$. Cell Biol., 146: 683-693.

Mizushima, S. and Nagata, S. 1990. pEF-BOS, a powerful mammalian expression vector. Nuc. Acid. Res., 18: 5322.

Morita, K., Sasaki, H., Furuse, M., and Tsukita, S. 1999. Endothelial claudin: claudin-5/TMVCF constitutes tight junction strands in endothelial cells. J. Cell Biol., 147: 185-194.

Rubin, L.L., Hall, D, E., Porter, S., Barbu, K., Cannon, C., Horner, H.C., Janatpour, M., Liaw, C.W., Manning, K., Morales, J., Tanner, L.I., Tomaselli, K.J., and Bard, F. 1991. A cell culture model of the bloodbrain barrier. J. Cell Biol., 115: 1725-1735.

Saito, S-Y., Watanabe, S., Ozaki, H., Fusetani, N., and Karaki, H. 1994. Mycalolide B, a novel actin depolymerizing agent. J. Biol. Chem., 269: 
$29710-29714$.

Saitou, M., Ando-Akatsuka, Y., Itoh, M., Furuse, M., Inazawa, J., Fujimoto, K., and Tsukita, S. 1997. Mammalian occludin in epithelial cells: its expression and subcellular distribution. Eur. J. Cell Biol., 73: 222-231.

Satoh, H., Zhong, Y., Isomura, H., Saitoh, M., Enomoto, K., Sawada, N., and Mori, M. 1996. Localization of $7 \mathrm{H} 6$ tight junction-associated paracellular barrier function against ions, large molecules, and cancer cells. Exp. Cell Res., 222: 269-274.

Schulze, C., Smales, C., Rubin, L.L., and Staddon, J.M. 1997. Lysophos- phatidic acid increases tight junction permeability in cultured brain endothelial cells. J. Neurochem., 68: 991-1000.

Takakuwa, R., Kokai, Y., Kojima, T., Akatsuka, T., Tobioka, H., Sawada, N., and Mori, M. 2000. Uncoupling of gate and fence functions of MDCK cells by the actin-depolymerizing reagent mycalolide B. Exp. Cell Res., 257: 238-244.

(Received for publication, April 16, 2001

and accepted, April 18, 2001) 